\title{
Search for Large-scale Anisotropy on Arrival Directions of Ultra-high- energy Cosmic Rays Observed with the Telescope Array Experiment
}

\section{$\operatorname{AUTHOR}(\mathrm{S}):$}

Abbasi, R. U.; Abe, M.; Abu-Zayyad, T.; Allen, M.; Azuma, R.; Barcikowski, E.; Belz, J. W.; ... Yosei, M.; Zhezher, Y.; Zundel, Z.

\section{CITATION:}

Abbasi, R. U.... [et al]. Search for Large-scale Anisotropy on Arrival Directions of Ultra-highenergy Cosmic Rays Observed with the Telescope Array Experiment. The Astrophysical Journal Letters 2020, 898(2): L28.

\section{ISSUE DATE:}

2020-08-01

URL:

http://hdl.handle.net/2433/253550

\section{RIGHT:}

This is the Accepted Manuscript version of an article accepted for publication in The Astrophysical Journal Letters. IOP Publishing Ltd is not responsible for any errors or omissions in this version of the manuscript or any version derived from it. The Version of Record is available online at https://doi.org/10.3847/2041-8213/aba0bc;; The full-text file will be made open to the public on 27 July 2021 in accordance with publisher's 'Terms and Conditions for Self-Archiving'.; $こ の$ 論文は出版社版でありません。引用の際には出版社版をご確認ご利用ください。；This is not the published version.

Please cite only the published version. 
DRAFT VERSION JULY 28, 2020

Typeset using LATEX twocolumn style in AASTeX62

Search for Large-scale Anisotropy on Arrival Directions of Ultra-high-energy Cosmic Rays Observed with the Telescope Array Experiment

Telescope Array Collaboration

R.U. Abbasi, ${ }^{1}$ M. Abe, ${ }^{2}$ T. Abu-Zayyad,${ }^{3}$ M. Allen,${ }^{3}$ R. Azuma,${ }^{4}$ E. Barcikowski,${ }^{3}$ J.W. Belz,${ }^{3}$ D.R. Bergman, ${ }^{3}$ S.A. Blake, ${ }^{3}$ R. Cady,${ }^{3}$

B.G. Cheon, ${ }^{5}$ J. Chiba, ${ }^{6}$ M. Chikawa, ${ }^{7}$ A. Di Matteo, ${ }^{8, *}$ T. Fujil, ${ }^{9}$ K. Fujisue, ${ }^{7}$ K. Fujita, ${ }^{10}$ R. Fujiwara, ${ }^{10}$ M. Fukushima, ${ }^{7,11}$ G. Furlich, ${ }^{3}$

W. Hanlon, ${ }^{3}$ M. Hayashi ${ }^{12}$ N. Hayashida, ${ }^{13}$ K. Hibino, ${ }^{13}$ R. Higuchi,,${ }^{7}$ K. Honda,${ }^{14}$ D. Ikeda,${ }^{15}$ T. Inadomi,${ }^{16}$ N. Inoue, ${ }^{2}$ T. Ishit, ${ }^{14}$ R. Ishimori, ${ }^{4}$

H. Ito, ${ }^{17}$ D. Ivanov, ${ }^{3}$ H. Imakura, ${ }^{16}$ H.M. Jeong, ${ }^{18}$ S. Jeong, ${ }^{18}$ C.C.H. Jui, ${ }^{3}$ K. Kadota, ${ }^{19}$ F. Kakimoto, ${ }^{13}$ O. Kalashev, ${ }^{20}$ K. Kasahara, ${ }^{21}$

S. Kasami, ${ }^{22}$ H. Kawai, ${ }^{23}$ S. Kawakami, ${ }^{10}$ S. Kawana, ${ }^{2}$ K. Kawata,${ }^{7}$ E. Kido, ${ }^{7}$ H.B. Kim,,${ }^{5}$ J.H. Kim, ${ }^{10}$ J.H. Kim, ${ }^{3}$ M.H. Kim, ${ }^{18}$ S.W. Kim, ${ }^{18}$

S. Kishigami, ${ }^{10}$ V. Kuzmin,,${ }^{20}{ }^{\dagger}$ M. Kuznetsov, ${ }^{20,8}$ Y.J. Kwon, ${ }^{24}$ K.H. Lee, ${ }^{18}$ B. Lubsandorzhiev, ${ }^{20}$ J.P. Lundquist, ${ }^{3}$ K. Machida, ${ }^{14}$

H. Matsumiya, ${ }^{10}$ T. Matsuyama, ${ }^{10}$ J.N. Matthews, ${ }^{3}$ R. Mayta,${ }^{10}$ M. Minamino, ${ }^{10}$ K. Mukai,,${ }^{14}$ I. Myers, ${ }^{3}$ S. Nagataki, ${ }^{17}$ K. Nakai, ${ }^{10}$

R. Nakamura, ${ }^{16}$ T. Nakamura, ${ }^{25}$ Y. Nakamura, ${ }^{16}$ Y. Nakamura, ${ }^{16}$ T. Nonaka, ${ }^{7}$ H. Oda, ${ }^{10}$ S. Ogio, ${ }^{10,26}$ M. OhNishi, ${ }^{7}$ H. OhоKa, ${ }^{7}$ Y. OKu, ${ }^{22}$

T. Okuda,${ }^{27}$ Y. Omura,${ }^{10}$ M. Ono, ${ }^{17}$ R. Onogi, ${ }^{10}$ A. Oshima, ${ }^{10}$ S. Ozawa, ${ }^{28}$ I.H. Park, ${ }^{18}$ M.S. Pshirkov, ${ }^{20,29}$ J. Remington, ${ }^{3}$ D.C. Rodriguez, ${ }^{3}$

G. Rubtsov ${ }^{20}$ D. Ryu ${ }^{30}$ H. Sagawa, ${ }^{7}$ R. Sahara,${ }^{10}$ Y. Saito, ${ }^{16}$ N. Sakaki, ${ }^{7}$ T. Sako, ${ }^{7}$ N. Sakurai ${ }^{10}$ K. Sano, ${ }^{16}$ T. SeKi, ${ }^{16}$ K. Sekino, ${ }^{7}$

P.D. Shah, ${ }^{3}$ F. Shibata, ${ }^{14}$ T. Shibata, ${ }^{7}$ H. Shimodaira, ${ }^{7}$ B.K. Shin, ${ }^{30}$ H.S. Shin, ${ }^{7}$ J.D. Smith, ${ }^{3}$ P. Sokolsky, ${ }^{3}$ N. Sone, ${ }^{16}$ B.T. Stokes, ${ }^{3}$

T.A. Stroman, ${ }^{3}$ T. Suzawa, ${ }^{2}$ Y. Takagi,${ }^{10}$ Y. Takahashi, ${ }^{10}$ M. Takamura,${ }^{6}$ M. Takeda, ${ }^{7}$ R. Takeishi, ${ }^{18}$ A. Taketa, ${ }^{15}$ M. Takita, ${ }^{7}$ Y. Tameda, ${ }^{22}$

H. Tanaka, ${ }^{10}$ K. Tanaka, ${ }^{31}$ M. Tanaka, ${ }^{32}$ Y. Tanoue, ${ }^{10}$ S.B. Thomas, ${ }^{3}$ G.B. Thomson, ${ }^{3}$ P. Tinyakov,${ }^{20,8}{ }^{8}$ I. Tkachev,${ }^{20}$ H. Tokuno, ${ }^{4}$ T. Tomida ${ }^{16}$

S. Troitsky, ${ }^{20}$ Y. Tsunesada,,${ }^{10,26}$ Y. Uchinori, ${ }^{33}$ S. Udo,${ }^{13}$ T. Uehama, ${ }^{16}$ F. Urban, ${ }^{34}$ T. Wong,${ }^{3}$ K. Yada,${ }^{7}$ M. Yamamoto, ${ }^{16}$ K. Yamazaki, ${ }^{13}$ J. Yang, ${ }^{35}$ K. Yashiro, ${ }^{6}$ M. Yosei, ${ }^{22}$ Y. ZheZher, ${ }^{7,20}$ AND Z. Zundel ${ }^{3}$

${ }^{1}$ Department of Physics, Loyola University Chicago, Chicago, Illinois, USA

${ }^{2}$ The Graduate School of Science and Engineering, Saitama University, Saitama, Saitama, Japan

${ }^{3}$ High Energy Astrophysics Institute and Department of Physics and Astronomy, University of Utah, Salt Lake City, Utah, USA

${ }^{4}$ Graduate School of Science and Engineering, Tokyo Institute of Technology, Meguro, Tokyo, Japan

${ }^{5}$ Department of Physics and The Research Institute of Natural Science, Hanyang University, Seongdong-gu, Seoul, Korea

${ }^{6}$ Department of Physics, Tokyo University of Science, Noda, Chiba, Japan

${ }^{7}$ Institute for Cosmic Ray Research, University of Tokyo, Kashiwa, Chiba, Japan

${ }^{8}$ Service de Physique Théorique, Université Libre de Bruxelles, Brussels, Belgium

${ }^{9}$ The Hakubi Center for Advanced Research and Graduate School of Science, Kyoto University, Kitashirakawa-Oiwakecho, Sakyo-ku, Kyoto, Japan

${ }^{10}$ Graduate School of Science, Osaka City University, Osaka, Osaka, Japan

${ }^{11}$ Kavli Institute for the Physics and Mathematics of the Universe (WPI), Todai Institutes for Advanced Study, University of Tokyo, Kashiwa, Chiba, Japan

${ }^{12}$ Information Engineering Graduate School of Science and Technology, Shinshu University, Nagano, Nagano, Japan

${ }^{13}$ Faculty of Engineering, Kanagawa University, Yokohama, Kanagawa, Japan

${ }^{14}$ Interdisciplinary Graduate School of Medicine and Engineering, University of Yamanashi, Kofu, Yamanashi, Japan

${ }^{15}$ Earthquake Research Institute, University of Tokyo, Bunkyo-ku, Tokyo, Japan

${ }^{16}$ Academic Assembly School of Science and Technology Institute of Engineering, Shinshu University, Nagano, Nagano, Japan

${ }^{17}$ Astrophysical Big Bang Laboratory, RIKEN, Wako, Saitama, Japan

${ }^{18}$ Department of Physics, Sungkyunkwan University, Jang-an-gu, Suwon, Korea

${ }^{19}$ Department of Physics, Tokyo City University, Setagaya-ku, Tokyo, Japan

${ }^{20}$ Institute for Nuclear Research of the Russian Academy of Sciences, Moscow, Russia

${ }^{21}$ Faculty of Systems Engineering and Science, Shibaura Institute of Technology, Minato-ku, Tokyo, Japan

${ }^{22}$ Department of Engineering Science, Faculty of Engineering, Osaka Electro-Communication University, Neyagawa-shi, Osaka, Japan

${ }^{23}$ Department of Physics, Chiba University, Chiba, Chiba, Japan

${ }^{24}$ Department of Physics, Yonsei University, Seodaemun-gu, Seoul, Korea

${ }^{25}$ Faculty of Science, Kochi University, Kochi, Kochi, Japan

${ }^{26}$ Nambu Yoichiro Institute of Theoretical and Experimental Physics, Osaka City University, Osaka, Osaka, Japan

${ }^{27}$ Department of Physical Sciences, Ritsumeikan University, Kusatsu, Shiga, Japan

${ }^{28}$ Quantum ICT Advanced Development Center, National Institute for Information and Communications Technology, Koganei, Tokyo, Japan

${ }^{29}$ Sternberg Astronomical Institute, Moscow M.V. Lomonosov State University, Moscow, Russia

${ }^{30}$ Department of Physics, School of Natural Sciences, Ulsan National Institute of Science and Technology, UNIST-gil, Ulsan, Korea

${ }^{31}$ Graduate School of Information Sciences, Hiroshima City University, Hiroshima, Hiroshima, Japan

${ }^{32}$ Institute of Particle and Nuclear Studies, KEK, Tsukuba, Ibaraki, Japan 


\footnotetext{
${ }^{33}$ Department of Research Planning and Promotion, Quantum Medical Science Directorate, National Institutes for Quantum and Radiological Science and Technology, Chiba, Chiba, Japan

${ }^{34}$ CEICO, Institute of Physics, Czech Academy of Sciences, Prague, Czech Republic

${ }^{35}$ Department of Physics and Institute for the Early Universe, Ewha Womans University, Seodaaemun-gu, Seoul, Korea
}

\begin{abstract}
Motivated by the detection of a significant dipole structure in the arrival directions of ultra-high-energy cosmic rays above $8 \mathrm{EeV}$ reported by the Pierre Auger Observatory (Auger), we search for a large-scale anisotropy using data collected with the surface detector array of the Telescope Array Experiment (TA). With 11 years of TA data, a dipole structure in a projection of the right ascension is fitted with an amplitude of $3.3 \pm 1.9 \%$ and a phase of $131^{\circ} \pm 33^{\circ}$. The corresponding $99 \%$ confidence-level upper limit on the amplitude is $7.3 \%$. At the current level of statistics, the fitted result is compatible with both an isotropic distribution and the dipole structure reported by Auger.
\end{abstract}

Keywords: astroparticle physics — ultra-high-energy comic ray — large-scale anisotropy

\section{INTRODUCTION}

Clarifying the origin and nature of the ultra-high-energy cosmic rays (UHECRs) has been a decades-long endeavor (Dawson et al. 2017; Alves Batista et al. 2019). There are a number of challenges in identifying sources, including the uncertainty in the chemical composition of cosmic rays with energies above $10 \mathrm{EeV}\left(\equiv 10^{19} \mathrm{eV}\right)$, and existence of galactic and extragalactic magnetic fields that scramble directional information.

The origin of UHECRs above $50 \mathrm{EeV}$ would be significantly restricted to nearby sources due to interactions with the cosmic microwave background radiation via pion production for protons or via photo-disintegration processes for heavier nuclei, known as the GZK cutoff (Greisen 1966; Zatsepin \& Kuzmin 1966). With small deflections of UHECRs by galactic and intergalactic magnetic fields, $\sim 5^{\circ} Z(E / 50 \mathrm{EeV})^{-1}$ where $Z$ is the charge of nuclei (Bray \& Scaife 2018), a small-intermediate scale anisotropy ${ }^{1}$ is predicted at the highest energies. On the other hand, the distance to sources and deflections by magnetic fields are enlarged at lower energies around $10 \mathrm{EeV}$, resulting in a large-scale anisotropy, which can be approximated as a dipole (Harari et al. 2015; di Matteo \& Tinyakov 2018; Eichmann \& Winchen 2020). Full-sky anisotropy measurements from both hemispheres are also essential to understand the large-scale anisotropy.

The two largest observatories, the Telescope Array Experiment (TA) (Tokuno et al. 2012; Abu-Zayyad et al. 2013a) and Pierre Auger Observatory (Auger) (Aab et al. 2015), are currently in operation and observing UHECRs from the Northern and Southern hemispheres, respectively. There

\footnotetext{
${ }^{*}$ Currently at INFN, sezione di Torino, Turin, Italy

$\dagger$ Deceased

${ }^{1}$ In this research field, we define a small angular scale anisotropy as less than $5^{\circ}$, intermediate scale as $10^{\circ}$ to $35^{\circ}$ and large scale as $>40^{\circ}$.
}

are indications of a number of intriguing intermediate-scale anisotropies at the highest energies $(E>30 \mathrm{EeV})$, such as the hotspot reported by TA (Abbasi et al. 2014) and the result of the flux pattern correlation analysis from Auger (Aab et al. 2018a) and TA (Abbasi et al. 2018). However, no conclusive results are reported and the origins of UHECRs are still unknown.

In 2017 the Pierre Auger Collaboration reported the observation of a significant large-scale anisotropy in the arrival directions of cosmic rays above $8 \mathrm{EeV}$, indicating an obvious dipole structure of $4.7 \%$ amplitude in a projection of the right ascension with a 5.2 $\sigma$ significance (Aab et al. 2017). An enhancement of the dipole amplitude above $4 \mathrm{EeV}$ and results down to $0.03 \mathrm{EeV}$ are also reported (Aab et al. 2018b, 2020) These results are consistent with an extragalactic origin of UHECRs. The dipole anisotropy of right ascension over a broad energy range is reported by a variety of experiments, as summarized in (Mollerach \& Roulet 2018).

In this letter we investigate the existence of a dipole structure using TA data. TA detects UHECRs from the Northern hemisphere, providing us with an independent check of the large-scale anisotropy reported by Auger.

\section{TELESCOPE ARRAY EXPERIMENT}

TA is the largest cosmic-ray detector in the Northern hemisphere, located near the city of Delta, Utah, USA $\left(39.30^{\circ}\right.$ North and $112.91^{\circ}$ West at $\sim 1400 \mathrm{~m}$ above sea level) (AbuZayyad et al. 2013a). The surface detector array (SD) consists of 507 plastic scintillators of $3 \mathrm{~m}^{2}$ area deployed in a square grid with a $1.2 \mathrm{~km}$ spacing. The total effective area is approximately $700 \mathrm{~km}^{2}$. Additional surface detectors, designed to provide a fourfold increase in effective area, named TAx4, are now being deployed and partly in operation (Kido et al. 2019). The TA SD is overlooked by fluorescence detectors, which are used for determination of the calorimetric energy of an air shower from energy deposited in the atmosphere during its development (Tokuno et al. 2012). 
The arrival direction of UHECR as measured by the TA $\mathrm{SD}$ is determined from the relative difference in arrival time of the shower front at each surface detector (which are timesynchronized using GPS modules). The energy estimator of the TA SD is the particle density measured at a distance of $800 \mathrm{~m}$ from the air shower axis, called $S_{800}$. The $S_{800}$ parameter is converted to the primary energy as a function of zenith angle based on a Monte Carlo simulation using the CORSIKA software package (Heck et al. 1998). The obtained energy is calibrated to the calorimetric energy measured by the fluorescence detectors using a scaling factor of 1/1.27 (AbuZayyad et al. 2013b). The typical resolution of the TA SD is $1.0^{\circ} \sim 1.5^{\circ}$ in arrival direction and $10 \% \sim 15 \%$ in primary energy (Abu-Zayyad et al. 2013b), and the systematic uncertainty in the energy scale is quoted as $21 \%$ (Abbasi et al. 2016).

\section{DATA-SET AND METHODOLOGY}

TA SD data recorded over 11 years from May 2008 to May 2019 were analyzed for a study of the large-scale anisotropy. To avoid potential penalties from scanning, we use an a priori energy threshold of $8.8 \mathrm{EeV}$, equivalent to $8 \mathrm{EeV}$ used by Auger, taking into account the $10 \%$ energy scale difference between TA and Auger calibrated from a common hardening feature of both energy spectra around $5 \mathrm{EeV}$ (Verzi et al. 2017). There were 6032 events surviving this cut above a primary energy of $8.8 \mathrm{EeV}$, with zenith angles below $55^{\circ}$ and the same quality cuts used in the TA spectrum analysis (AbuZayyad et al. 2013b). In this data-set, TA SD is capable of measuring UHECRs injected in a declination band from $-15^{\circ}$ to $90^{\circ}$.

As the trigger efficiency of the TA SD at $8.8 \mathrm{EeV}$ is not $100 \%$, the obtained right ascension distribution is compared with the expected distribution assuming an isotropic UHECR sky from a time-dependent Monte Carlo (MC) simulation including calibration constants, live time and dead time of each surface detector station, and TA SD trigger efficiencies. A sidereal time distribution (366.25 cycles/year) of the simulation has a small amplitude of $0.4 \%$. The residual intensity is defined as $\left(N_{\text {obs }}-N_{\exp }\right) / N_{\text {exp }}$, where $N_{\text {obs }}$ is the number of events observed by TA and $N_{\text {exp }}$ is the expected number estimated from the simulation. The number of total events in the MC simulation is normalized to the number of events in the TA data above $8.8 \mathrm{EeV}$.

We also check for possible declination band dependence in common with Auger with $\delta<24.8^{\circ}$ where $\delta$ is the declination, and a higher declination band with $\delta \geq 24.8^{\circ}$. These declination bands were defined in (Verzi et al. 2017).

\section{RESULTS}

\subsection{Dipole structure in right ascension}

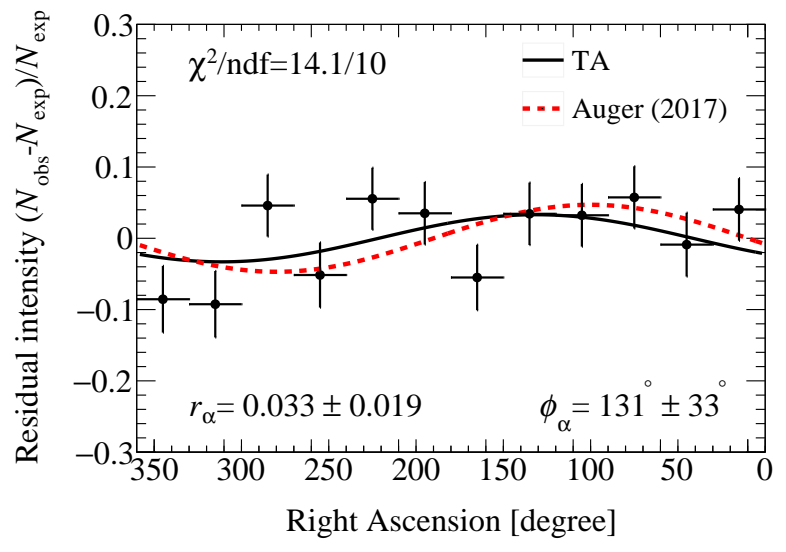

Figure 1. Residual intensities of UHECRs with energies above 8.8 EeV observed with 11 years of TA data as a function of the right ascension. The black curve is the TA fitted dipole result and the red dashed curve is the Auger reported dipole result.

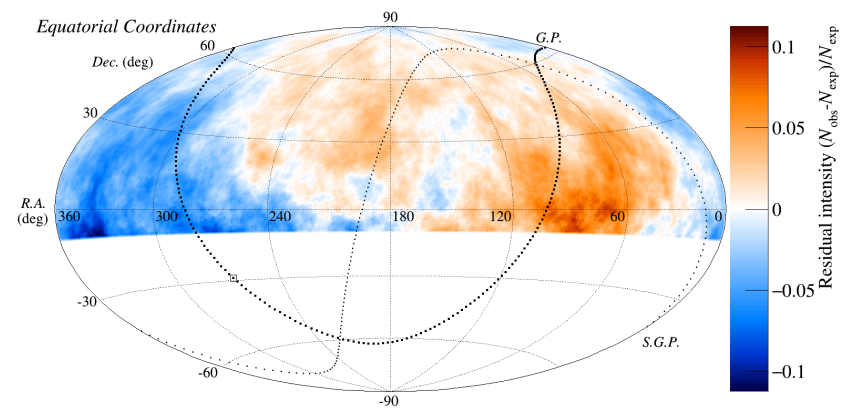

(a) Equatorial coordinates

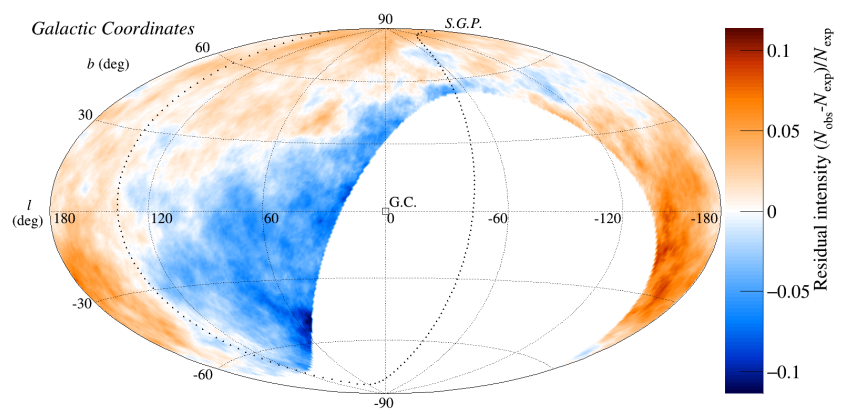

(b) Galactic coordinates

Figure 2. Sky map of residual intensities between TA data and an isotropic distribution shown in the Equatorial and the Galactic coordinates. The arrival directions are oversampled with a $45^{\circ}$ radius cylindrical function. The galactic plane (G.P.) and the super-galactic plane (S.G.P.) are shown as thick and thin dotted curves, respectively. The galactic center (G.C.) is indicated by the open square.

Figure 1 shows the residual intensity as a function of right ascension between 11 years of TA SD data and the isotropic expectation calculated from the $\mathrm{MC}$ simulation. The residual intensity is fitted to $r_{\alpha} \cos \left(x-\phi_{\alpha}\right)$, where $r_{\alpha}$ is the amplitude of the dipole and $\phi_{\alpha}$ is the phase. The obtained dipole struc- 


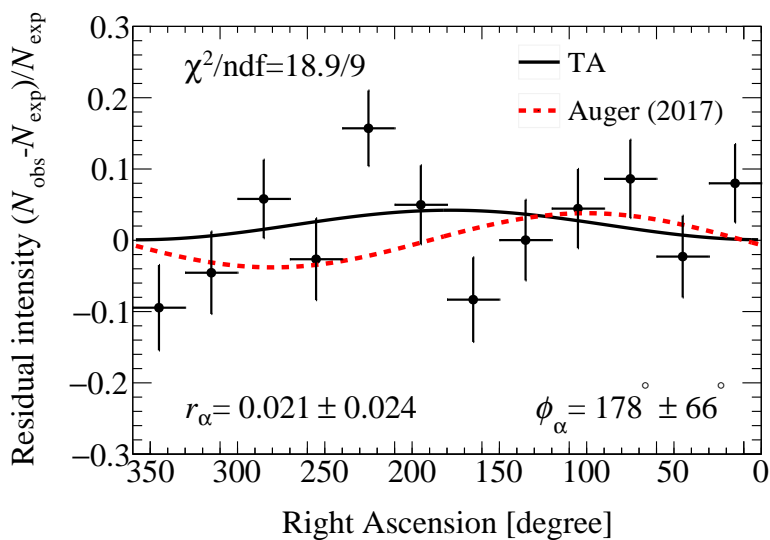

(a) Higher declination band, $\delta \geq 24.8^{\circ}$

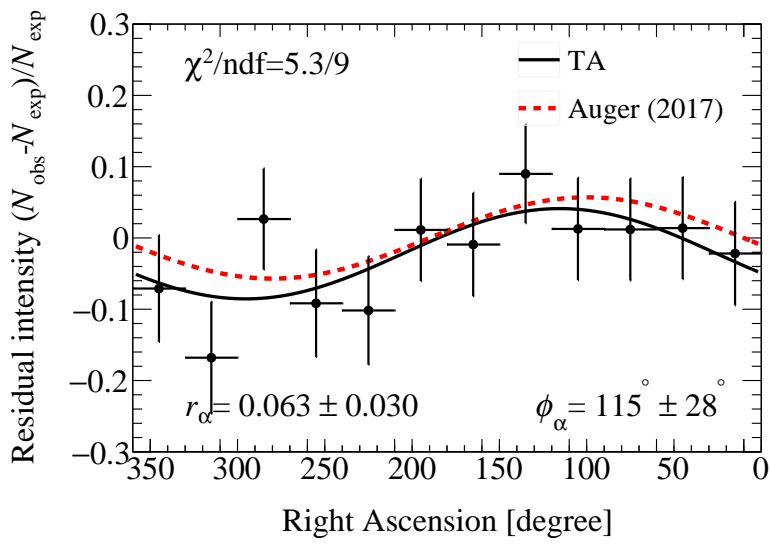

(b) Common declination band with Auger, $\delta<24.8^{\circ}$

Figure 3. Residual intensity in right ascension in (a) the higher declination band, $\delta \geq 24.8^{\circ}$, and (b) the common declination band with Auger, $\delta<24.8^{\circ}$. The solid black curve is the fit to the TA SD data, and the red dashed curve is the expected dipole structure from the Auger result, 3.8\% amplitude in the higher band and 5.7\% amplitude in the common band.

ture has an amplitude of $3.3 \pm 1.9 \%$ with a phase of $131^{\circ} \pm 33^{\circ}$. The TA SD data points are also compared with an isotropic distribution and the dipole structure reported from Auger, which has a $4.7 \%$ amplitude with a phase of $100^{\circ}$. The $\chi^{2} /$ ndf against the isotropic distribution is $17.1 / 12$ with a probability of $14 \%$, and that against the Auger dipole is $15.9 / 12$ with a probability of $20 \%$. With current statistics, the obtained TA SD result is consistent with both hypotheses.

Since there is no significant dipole structure, an upper limit is evaluated for a chance probability of $1 \%$ that a fluctuation of an isotropic source distribution would yield an amplitude greater than the measured value. The obtained $99 \%$ confidence-level upper-limit on amplitude is $7.3 \%$ for TA data above $8.8 \mathrm{EeV}$.

\subsection{Systematic uncertainty}

When we change to an assumption of $100 \%$ trigger efficiency and of uniform exposure to calculate the expected number of events from each right ascension bin, we have a dipole shifted by the amplitude of $-0.4 \%$ and the phase of $+5^{\circ}$. This gives a possible spurious dipole due to timedependent detector conditions.

A modulation related to the atmospheric condition is seen in the TA SD data. When an empirical energy correction as a function of the atmospheric density at the ground is applied, the fitted dipole result is changed by $-1.1 \%$ in amplitude and $-23^{\circ}$ in phase. The correction method related to the atmospheric condition is being investigated and validated. These systematic uncertainties are smaller than the current statistical uncertainty of $1.9 \%$ in amplitude in the TA SD data.

The anti-sidereal time distribution ( 364.25 cycles/year) is also tested for a systematic study. The fitted amplitude is $1.8 \pm 1.8 \%$, being consistent with a uniform distribution.

\subsection{Residual intensity sky map}

To investigate the dipole structure, the residual-intensity sky map is calculated with a $45^{\circ}$ radius cylindrical smoothing function, usually known as a top-hat function, applied to the arrival directions of UHECRs as used in Auger (Aab et al. 2017). Figure 2 shows the residual-intensity sky map of UHECRs measured by TA with energies above $8.8 \mathrm{EeV}$ in equatorial and galactic coordinates. Even with our limited statistics, a similar dipole structure is seen in the common declination $\delta<24.8^{\circ}$ band shared with Auger.

\subsection{Declination dependence}

Figure 3 shows the residual intensities and fitted results in the higher declination band above $24.8^{\circ}$ and the declination band in common with Auger below 24.8 . If we assume the three-dimensional dipole structure reported by Auger, with $6.5 \%$ amplitude at $100^{\circ}$ in right ascension and $-24^{\circ}$ in declination (Aab et al. 2017), the expected amplitudes are evaluated to be $3.8 \%$ in the higher declination band and 5.7\% in the common declination band with Auger. These expectations are indicated by the curves in the plots of Figure 3 . An offset parameter is added to the fitting function as we divide the data into two sets without a re-normalization in each dataset.

The obtained results are still inconclusive. The fitted function in the higher declination band has an amplitude of $2.1 \pm 2.4 \%$ at $178^{\circ} \pm 66^{\circ}$. The $\chi^{2} /$ ndf against the isotropic distribution is $21.2 / 12$ with a probability of $5 \%$, and that against the reported dipole from Auger is 23.1/12 with a probability of $3 \%$. The dipole structure in the common declination band with Auger is fitted by an amplitude of $6.3 \pm 3.0 \%$ at $115^{\circ} \pm 28^{\circ}$, which is consistent with the one reported by Auger. Also the $\chi^{2} / \mathrm{ndf}$ against the isotropic distribution is $10.8 / 12$ with a probability of $55 \%$, and that against the Auger 
SEARCH FOR LARGE-SCALE ANISOTROPY ON ARRIVAL DIRECTIONS OF UHECRS OBSERVED WITH TA

\begin{tabular}{l|ccccc} 
& $E_{\text {med }}[\mathrm{EeV}]$ & $N$ & $r_{\alpha}[\%]$ & $\phi_{\alpha}\left[^{\circ}\right]$ & $r_{\alpha}^{\mathrm{UL}}[\%]$ \\
\hline All & 13.0 & 6032 & $3.3 \pm 1.9$ & $131 \pm 33$ & 7.3 \\
$\delta \geq 24.8^{\circ}$ & - & 3778 & $2.1 \pm 2.4$ & $178 \pm 66$ & 6.7 \\
$\delta<24.8^{\circ}$ & - & 2254 & $6.3 \pm 3.0$ & $115 \pm 28$ & 12.9
\end{tabular}

Table 1. Summary of the median energy, number of events, the dipole fitted results and the corresponding upper limits (UL) amplitude at $99 \%$ confidence level in all bands, in the higher declination band, $\delta \geq 24.8^{\circ}$, and in the common declination band with Auger, $\delta$ $<24.8^{\circ}$.

result is $6.8 / 12$ with a probability of $80 \%$. The fitted parameters and corresponding upper limits at $99 \%$ confidence level in these bands are summarized in Table 1,

\section{DISCUSSION}

Due to the limited statistics with the TA data, the obtained amplitudes are compatible with an isotropic distribution at a $2 \sigma$ significance level. However, focusing on the common declination band with Auger and the sky map of residual intensities in Figure 2, the fitted phase is apart from the galactic center of $266^{\circ}$ in right ascension, supporting an extragalactic origin of UHECRs reported from Auger. In the higher declination band, probabilities of 5\% against the isotropic distribution and of 3\% against the Auger reported dipole might be influenced by additional components of the anisotropy, possibly related to local sources and magnetic fields.

Further data-taking with TA and TA $\times 4$ will be essential to differentiate between the isotropic and the dipole structure hypotheses, and capable to investigate an energy dependence of the dipole amplitude reported from Auger (Aab et al. 2018b). Continuing the full-sky anisotropy searches from both hemispheres is of the upmost importance to clarify the origin and nature of UHECRs.

\section{CONCLUSIONS}

We report on a follow-up search for the dipole structure reported by Auger using 11 years of TA SD data from the Northern sky. We see results consistent with both an isotropic source distribution and the dipole structure reported by Auger. Therefore we have evaluated a $99 \%$ confidencelevel upper limit of $r_{\alpha}^{\mathrm{UL}}=7.3 \%$ above $8.8 \mathrm{EeV}$ on the amplitude of a dipole structure in a projection of the right ascension. Although the residual intensity sky map shows a similar dipole structure to Auger, further statistics from TA are required to distinguish the two hypotheses. Further data collection by TA and the on-going upgrade of TA $\times 4$ will be essential for further studies.

\section{ACKNOWLEDGMENTS}

The Telescope Array experiment is supported by the Japan Society for the Promotion of Science(JSPS) through Grantsin-Aid for Priority Area 431, for Specially Promoted Research JP21000002, for Scientific Research (S) JP19104006, for Specially Promoted Research JP15H05693, for Scientific Research (S) JP15H05741, for Science Research (A) JP18H03705, for Young Scientists (A) JPH26707011, and for Fostering Joint International Research (B) JP19KK0074, by the joint research program of the Institute for Cosmic Ray Research (ICRR), The University of Tokyo; by the U.S. National Science Foundation awards PHY-0601915, PHY1404495, PHY-1404502, and PHY-1607727; by the National Research Foundation of Korea (2016R1A2B4014967, 2016R1A5A1013277, 2017K1A4A3015188, 2017R1A2A1A05071429) ; by the Russian Academy of Sciences, RFBR grant 20-0200625a (INR), IISN project No. 4.4502.13, and Belgian Science Policy under IUAP VII/37 (ULB). The foundations of Dr. Ezekiel R. and Edna Wattis Dumke, Willard L. Eccles, and George S. and Dolores Doré Eccles all helped with generous donations. The State of Utah supported the project through its Economic Development Board, and the University of Utah through the Office of the Vice President for Research. The experimental site became available through the cooperation of the Utah School and Institutional Trust Lands Administration (SITLA), U.S. Bureau of Land Management (BLM), and the U.S. Air Force. We appreciate the assistance of the State of Utah and Fillmore offices of the BLM in crafting the Plan of Development for the site. Patrick Shea assisted the collaboration with valuable advice on a variety of topics. The people and the officials of Millard County, Utah have been a source of steadfast and warm support for our work which we greatly appreciate. We are indebted to the Millard County Road Department for their efforts to maintain and clear the roads which get us to our sites. We gratefully acknowledge the contribution from the technical staffs of our home institutions. An allocation of computer time from the Center for High Performance Computing at the University of Utah is gratefully acknowledged.

T.F. greatly appreciate the support of the Hakubi Center for Advanced Research, Kyoto University.

\section{REFERENCES}

Aab, A., et al. 2015, Nucl. Instrum. Meth., A798, 172,

doi: 10.1016/j.nima.2015.06.058

—. 2017, Science, 357, 1266, doi: 10.1126/science.aan4338
—. 2018a, Astrophys. J., 853, L29, doi: 10.3847/2041-8213/aaa66d

—. 2018b, Astrophys. J., 868, 4, doi: 10.3847/1538-4357/aae689

—. 2020, Astrophys. J., 891, 142, doi: 10.3847/1538-4357/ab7236 
Abbasi, R. U., et al. 2014, Astrophys. J., 790, L21, doi: 10.1088/2041-8205/790/2/L21

—. 2016, Astropart. Phys., 80, 131, doi: 10.1016/j.astropartphys.2016.04.002

—. 2018, Astrophys. J., 867, L27, doi: 10.3847/2041-8213/aaebf9

Abu-Zayyad, T., et al. 2013a, Nucl. Instrum. Meth., A689, 87, doi: 10.1016/j.nima.2012.05.079

—. 2013b, Astrophys. J., 768, L1, doi: 10.1088/2041-8205/768/1/L1

Alves Batista, R., et al. 2019, Front. Astron. Space Sci., 6, 23, doi: 10.3389/fspas.2019.00023

Bray, J., \& Scaife, A. 2018, Astrophys. J., 861, 3, doi: $10.3847 / 1538-4357 /$ aac777

Dawson, B. R., Fukushima, M., \& Sokolsky, P. 2017, PTEP, 2017, 12A101, doi: 10.1093/ptep/ptx054

di Matteo, A., \& Tinyakov, P. 2018, Mon. Not. Roy. Astron. Soc., 476, 715, doi: 10.1093/mnras/sty277

Eichmann, B., \& Winchen, T. 2020, JCAP, 04, 047, doi: 10.1088/1475-7516/2020/04/047
Greisen, K. 1966, Phys.Rev.Lett., 16, 748, doi: 10.1103/PhysRevLett.16.748

Harari, D., Mollerach, S., \& Roulet, E. 2015, Phys. Rev. D, 92, 063014, doi: 10.1103/PhysRevD.92.063014

Heck, D., Knapp, J., Capdevielle, J. N., Schatz, G., \& Thouw, T. 1998, Forschungszentrum Karlsruhe Report, FZKA-6019

Kido, E., et al. 2019, EPJ Web Conf., 210, 06001, doi: 10.1051/epjconf/201921006001

Mollerach, S., \& Roulet, E. 2018, Prog. Part. Nucl. Phys., 98, 85, doi: 10.1016/j.ppnp.2017.10.002

Tokuno, H., et al. 2012, Nucl. Instrum. Meth., A676, 54, doi: 10.1016/j.nima.2012.02.044

Verzi, V., Ivanov, D., \& Tsunesada, Y. 2017, PTEP, 2017, 12A103, doi: $10.1093 /$ ptep/ptx082

Zatsepin, G., \& Kuzmin, V. 1966, JETP Lett., 4, 78 